\title{
FACTORES DE RIESGO CARDIOVASCULAR
}

\section{CARDIOVASCULAR RISK FACTORS}

Pág. 6,9
Recibido: 24 Enero 2020

Aceptado: 26 Enero 2020

Daniel Chevez Elizondo, Kristel Alfaro Amador, Fabio Salas Ureña, Alison Robledo Guzmán, Ernesto Lubker Canales, María Alfaro Vellanero. Estudiante de la carrera de Microbiología y Química Clínica, Universidad de Ciencias Médicas (UCIMED), Decanatura de Microbiología, San José, Costa Rica.

\section{Resumen}

Las enfermedades cardiovasculares son la principal causa de muerte a nivel mundial, cada año mueren más personas por enfermedades cardiovasculares que por cualquier otra causa de muerte. En Costa Rica, las enfermedades cardiovasculares constituyen una de las causas más comunes en la tasa de mortalidad. El riesgo cardiovascular se define como la probabilidad de padecer un evento cardiovascular en un determinado período. Por otro lado, los factores de riesgo cardiovasculares son aquellos signos biológicos o hábitos adquiridos que se presentan con mayor frecuencia en los pacientes con alguna enfermedad cardiovascular (hipertensión arterial, cardiopatía coronaria, enfermedad cerebrovascular, entre otras). Las enfermedades cardiovasculares tienen un origen multifactorial, por lo tanto, un factor de riesgo debe ser considerado en el contexto de los otros. Los factores de riesgo cardiovascular se dividen en 2 grandes grupos: no modificables como la edad, el sexo y los antecedentes familiares $y$, modificables, donde se incluye hipercolesterolemia, tabaquismo, diabetes, hipertensión arterial, obesidad y sedentarismo.

Palabras clave: enfermedades cardiovasculares, riesgo cardiovascular, factores de riesgo cardiovasculares.

\section{Abstract}

Cardiovascular diseases are the leading cause of death worldwide; more people die each year from cardiovascular disease than from any other cause of death. In Costa Rica, cardiovascular diseases suffer from one of the most common causes in the mortality rate. Cardiovascular risk is defined as the probability of suffering a cardiovascular event in a given period. On the other hand, cardiovascular risk factors are those biological signs or acquired habits that occur most frequently in patients with any cardiovascular disease (arterial hypertension, coronary heart disease, cerebrovascular disease, among others). Cardiovascular diseases have a multifactorial origin; therefore, a risk factor must be considered in the context of others. Cardiovascular risk factors are divided into 2 large groups: nonmodifiable such as age, sex and family history, and modifiable, including hypercholesterolemia, smoking, diabetes, high blood pressure, obesity and sedentary lifestyle.

Keywords:cardiovascular diseases, cardiovascular risk, cardiovascular risk factors.

\section{Introducción:}

Según la OMS, las enfermedades cardiovasculares (ECV) es uno de los mayores problemas de salud pública en el mundo, siendo la primera causa de mortalidad al ocasionar 17 millones de muertes al año. Estudios afirman que cada año mueren más personas por ECV que por cualquier otra causa de muerte (Vega, et al. 2015). Las ECV se deben en su mayoría a eventos coronarios y accidentes 
cerebrovasculares. Se estima que este problema es mucho mayor en países en vía de desarrollo que en países desarrollados y se considera que millones de personas padecen de factores de riesgo que no son comúnmente diagnosticados, tales como hipertensión arterial, tabaquismo, diabetes, hipercolesterolemias y dieta inadecuada (Díaz, et al. 2007) (WHO. 2002).

En Costa Rica, las ECV han ido aumentando en las últimas décadas, siendo así una de las primeras causas en la tasa de mortalidad, superando al cáncer (Chaves, S., 2016). Estudios mencionan que las ECV más frecuentes en nuestro país son la enfermedad isquémica del corazón y la enfermedad cerebrovascular y afirman que estas patologías se encuentran distribuidas por todo el país de forma semejante (Castillo, et al., 2006). Enfermedades cardiovasculares.

Las enfermedades cardiovasculares son un conjunto de trastornos que afectan al corazón y a los vasos sanguíneos. Según la OMS se pueden clasificar en hipertensión arterial (presión alta), cardiopatía coronaria (infarto de miocardio), enfermedad cerebrovascular, enfermedad vascular periférica, insuficiencia cardíaca, cardiopatía reumática, cardiopatía congénita y miocardiopatías (OMS, 2019).

\section{Factores de riesgo cardiovascular}

Los factores de riesgo cardiovasculares son aquellos signos biológicos y hábitos adquiridos que se presentan con mayor frecuencia en los pacientes con una ECV en relación con la población general. Las ECV tiene un origen multifactorial, incluso, personas asintomáticas corren peligro si presentan 2 o más factores de riesgo. Los factores de riesgo cardiovascular se dividen en no modificables y modificables. Los no modificables son aquellos imposibles de cambiar, como edad, sexo y antecedentes familiares. Los modificables son los susceptibles al cambio, bien sea mejorando el estilo de vida o con terapia farmacológica, ejemplo: hipercolesterolemia, tabaquismo, diabetes, hipertensión arterial, obesidad y sedentarismo (Vega, et al., 2011) (Benegas, et al., 2006) (Manzur, et al., 2005) (Pearson, et al., 2002) (Rubinstein, et al., 2010).

Entre los factores de riesgo principales, el tabaquismo está implicado en el proceso crónico de la arteriosclerosis, debido a compuestos como la nicotina que actúa a niveles del organismo produciendo alteraciones fisiológicas y a su vez aumenta la presión arterial, generando hipertensión arterial (Giugno, et al., 2012). Otro factor principal es el hipercolesterolemia, en la cual hay demasiadas lipoproteínas de baja densidad (LDL o "colesterol malo") en la sangre, estas comienzan a acumularse sobre las paredes de las arterias formando una placa e iniciando así el proceso de una ECV denominada aterosclerosis (THI, 2019).

Actualmente, se están investigando nuevos factores de riesgo o factores de riesgo emergentes, como la lipoproteína a, la homocisteína, la proteína c reactiva, el fibrinógeno, el factor VII, la adiponectina y la interleucina 6 , entre otros (Musunuru, et al., 2010).

\section{Conclusión}

Como ya se mencionó anteriormente, las ECV son la causa más frecuente de muerte no sólo en Costa Rica, sino a nivel mundial. Se conocen los principales factores de riesgo que se presentan en las ECV, los más comunes son la hipertensión arterial, el hipercolesterolemia y el tabaquismo. Aunque se tenga una vida saludable, muchas de estas ECV pueden ser asintomáticas y finalmente causar la muerte. 


\section{Bibliografía}

Banegas, J. R., Villar, F., Graciani, A., \& Rodríguez Artalejo, F. (2006). Epidemiología de las enfermedades cardiovasculares en España. Revista Española de Cardiología Suplementos, 6(7), 3G-12G.

Castillo, L. M., Alvarado, A. T., \& Sánchez, M. I. (2006). Enfermedad cardiovascular en Costa Rica. Revista Costarricense de Salud Pública, 15(28), pp. 3-16.

Chaves, S. V. (2016). Etiología y epidemiología del cáncer en costa rica. Revista Médica de Costa Rica y Centroamérica, 83(618).

Díaz-Realpe, J. E., Muñoz-Martínez, J., \& SierraTorres, C. H. (2007). Factores de riesgo para enfermedad cardiovascular en trabajadores de una institución prestadora de servicios de salud, Colombia. Revista de salud pública, 9, pp. 64-75. Giugno, E. R., Trabaj, G. C., \& Cano, L. M. (2012). El Tabaco como Factor de Riesgo Cardiovascular. Obtenido de: http://www.fepreva.org/curso/6to_ curso/material/ut33.pdf

Manzur F, Arrieta CO. Estudio sociológico y del conocimiento de los factores de riesgo de las enfermedades cardiovasculares en la Costa Caribe Colombiana (Estudio Caribe). Rev Colomb Cardiol 2005; 12, pp.122-128.

Musunuru K, Kral BK, Blumenthal R, Fuster V, Campbell $\mathrm{C}$, et al. The use of high sensitivity C-reactive protein in clinical practice. (2008) Nat Clin Pract Cardiovasc Med [Online]. [citado 17 de octubre 2019]; 5(10) pp. 621-635. Available at: http://www.nature.com/nrcardio/journal/v5/n10/ full/ncpcardio1322.html

Organización Mundial de la Salud. (2019). ¿Qué son las enfermedades cardiovasculares? [online]
Available at: https://www.who.int/cardiovascular_ diseases/about_cvd/es/ [Accessed 18 Oct. 2019]. Pearson TA, Blair SN, Daniels SR, Eckel RH, Fair JM, Fortmann SP et al. (2002). AHA guidelines for primary prevention of cardiovascular disease and stroke. Circulation; 106: pp. 388-91.

Texas Heart Institute. (2019). Factores de riesgo cardiovascular | Texas Heart Institute. [online] Available at: https://www.texasheart.org/hearthealth/heart-information-center/topics/factoresde-riesgo-cardiovascular/ [Accessed 18 Oct. 2019].

Rubinstein A, Colantonio L, Bardach A, Caporale J, García Martí S, Kopitowski K, et al. (2010). Estimación de la carga de las enfermedades cardiovasculares atribuible a factores de riesgo modificables en Argentina. Rev Panam Salud Pública. 27(4) pp. 237-245.

Vega Abascal, J., Guimará Mosqueda, M. R., Garces Hernández, Y., García Bermúdez, Y., \& Vega Abascal, L. A. (2015). Proteína C reactiva de alta sensibilidad y riesgo de enfermedad cardiovascular. Correo Científico Médico, 19(2), pp. 190-201.

Vega Abascal, J., Guimará Mosqueda, M., \& Vega Abascal, L. (2011). Riesgo cardiovascular, una herramienta útil para la prevención de las enfermedades cardiovasculares. Revista Cubana de Medicina General Integral, 27(1), pp. 91-97.

World Health Organization. The World Health report 2002: reducing risks, promoting healthy life. Geneva, Switzerland: WHO; 2002. pp. 1-230.

\section{$\fallingdotseq \oplus \Theta \Theta$}


